\title{
Charge transport through a single-electron transistor with a mechanically oscillating island
}

\author{
N. M. Chtchelkatchev, ${ }^{1,2,3}$ W. Belzig, ${ }^{1}$ and C. Bruder $^{1}$ \\ ${ }^{1}$ Departement Physik und Astronomie, Universität Basel, Klingelbergstr. 82, 4056 Basel, Switzerland \\ ${ }^{2}$ L. D. Landau Institute for Theoretical Physics, Russian Academy of Sciences, 117940 Moscow, Russia \\ ${ }^{3}$ Institute for High Pressure Physics, Russian Academy of Sciences, Troitsk 142092, Moscow Region, Russia
}

(Received 9 August 2004; published 11 November 2004)

\begin{abstract}
We consider a single-electron transistor (SET) whose central island is a nanomechanical oscillator. The gate capacitance of the SET depends on the mechanical displacement, thus, the vibrations of the island may influence the transport properties. Harmonic oscillations of the island and thermal vibrations change the transport characteristics in different ways. The changes in the Coulomb blockade oscillations and in the current noise spectral density help to determine in what way the island oscillates, and allow to estimate the amplitude and the frequency of the oscillations.
\end{abstract}

DOI: 10.1103/PhysRevB.70.193305

PACS number(s): 73.23.Hk, 72.70.+m, 85.35.-p

The interplay of electric currents through nanostructures with mechanical degrees of freedom has attracted a lot of interest recently, both from the experimental and theoretical side. ${ }^{1-12}$ One of the central questions of this field of nanophysics is how the vibrations of the oscillating part of a nanodevice influence its transport properties and vice versa. A number of nanomechanical devices were investigated in the last years, e.g., so-called single-electron shuttles. ${ }^{3-8}$ On the theoretical side, it was shown recently that electric currents passing through a dirty nanowire can stimulate its vibrations. ${ }^{13}$ Indications for thermal vibrations of suspended single-wall nanotubes doubly clamped between two contacts were observed. ${ }^{14}$

The nanomechanical properties of single-electron transistors (SETs) are of particular interest. Natural candidates are SETs built from carbon nanotubes. For instance, it was shown that the equilibrium shape of a suspended nanotube studied as a function of a gate voltage shows features related to single-electron electronics, e.g., Coulomb "quantization" of the nanotube displacement. ${ }^{10}$

In this report, we discuss how vibrations of the central island of the SET change the current and the noise. We show that the transport characteristics of the SET differ for islands oscillating thermally or harmonically. The Coulomb blockade peaks are split for harmonic oscillations and are broadened by thermal oscillations. The current noise spectrum has a peak at the frequency of the island oscillations that reduces to a $\delta$ peak when the island oscillates harmonically.Therefore, measuring the transport properties of the SET can help to determine in what way the island oscillates, and to find the amplitude and frequency of the oscillations.

The system that we want to study-a SET with a mechanically oscillating island-is sketched in Fig. 1. We assume that the island is coupled to the left $(\mathrm{L})$ and right $(\mathrm{R})$ leads by tunnel junctions but can mechanically vibrate. A suspended nanotube is a possible experimental realization of this scenario. ${ }^{10}$

The charge of the island is coupled to the leads and the gate through the capacitances $C_{L}, C_{R}$, and $C_{g}(z) ; z$ is the transverse deviation of the island center from its equilibrium position. When the island oscillates, the gate capacitance changes with $z$, and therefore the transport properties of the SET change.

We assume that electronic transport through the SET can be described by sequential tunneling. In this case, it is governed by four tunneling rates: ${ }^{15-17}$ the rate for electrons to tunnel onto the central region from the left $\left(\Gamma_{n \rightarrow n+1}^{\mathrm{L}}\right)$ and right $\left(\Gamma_{n \rightarrow n+1}^{\mathrm{R}}\right)$ and the rates for electrons to tunnel off the central region. The rates can be calculated via Fermi's golden rule. The energy change corresponding to the first tunnel process is

$$
\begin{gathered}
\Delta E_{n \rightarrow n+1}^{\mathrm{L}}=e\left(V_{g}-V_{\mathrm{L}}\right)+\varepsilon_{n}, \\
\varepsilon_{n}=E_{c}\left[\frac{1}{2}+n+\frac{C_{\mathrm{R}}\left(V_{\mathrm{R}}-V_{g}\right)+C_{\mathrm{L}}\left(V_{\mathrm{L}}-V_{g}\right)}{e}\right],
\end{gathered}
$$

where $E_{c}=e^{2} / C_{\Sigma}$ and $C_{\Sigma}=C_{\mathrm{L}}+C_{\mathrm{R}}+C_{g}(z)$. Defining $\gamma(\epsilon)$ $\equiv-\epsilon /[1-\exp (\beta \epsilon)]$, the rates can be written as

$$
\Gamma_{n \rightarrow n+1}^{\mathrm{L}}(z)=\frac{1}{e^{2} R_{\mathrm{L}}} \gamma\left(\Delta E_{n \rightarrow n+1}^{\mathrm{L}}(z)\right),
$$

where $R_{\mathrm{L}}$ is the resistance of the left junction. The other rates can be written similarly. The charge state of the SET is characterized by the probability $\rho_{n}(t)$ to find $n$ excess electrons on the island. The time evolution of $\rho_{n}(t)$ is governed by the master equation ${ }^{15-18}$

$$
\frac{\partial \rho_{i}}{\partial t}=\sum_{a= \pm 1}\left(\Gamma_{i+a \rightarrow i} \rho_{i+a}-\Gamma_{i \rightarrow i+a} \rho_{i}\right)
$$

where $\Gamma_{i \rightarrow j}=\Gamma_{i \rightarrow j}^{\mathrm{L}}+\Gamma_{i \rightarrow j}^{\mathrm{R}}$. The current and all its cumulants can be expressed through $\Gamma$ and $\rho .{ }^{19}$

Equation (3) has to be supplemented with the equation describing the oscillations of the island. The electrostatic force that acts on the island is $f(z)=\partial_{z} C_{g}\left(V_{g}-\varphi\right)^{2} / 2$, where $\varphi=\left(C_{\mathrm{L}} V_{\mathrm{L}}+C_{\mathrm{R}} V_{\mathrm{R}}+C_{g} V_{G}+q\right) / C_{\Sigma}$ is the potential of the island, and $q=\Sigma_{n} \rho_{n} n e$ the average charge. The motion of the island can then be described by a Langevin equation 


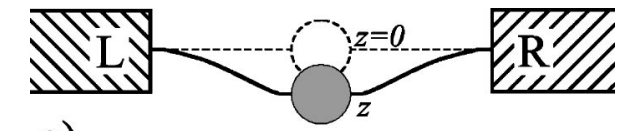

a)

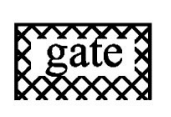

b)

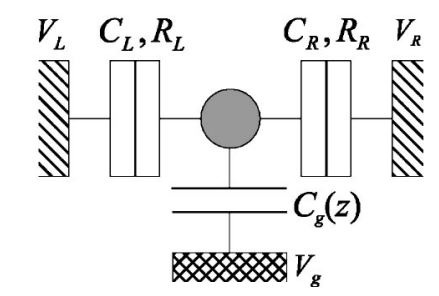

FIG. 1. (a) Sketch of a single-electron transistor (SET) with an oscillating island. The island is coupled to the left (L) and right (R) leads by tunnel junctions, and its capacitance to the gate $C_{g}(z)$ depends on the coordinate $z$ that measures the deviation of the island from its equilibrium position. (b) Equivalent circuit of the device.

$$
\ddot{z}+\eta \dot{z}+\omega_{0}^{2} z=y+[f(z)-f(0)] / m .
$$

Here $m$ is the island mass, $\eta \sim \omega_{0} / Q$ where $Q$ is the quality factor, and $y$ is a random force simulating the interaction with the thermal bath correlated as $\left\langle y(t) y\left(t^{\prime}\right)\right\rangle=2 \eta m T \delta(t$ $\left.-t^{\prime}\right) .{ }^{20}$ Typically $Q \sim 10^{3}-10^{41}$ and $\eta \ll \omega_{0}$. If the island is a nanotube, $C_{g}$ is in general a functional of the deviation $z(x)$, where $x$ is the coordinate along the nanotube. ${ }^{10}$ The rates depend only on integral quantities like $\int_{0}^{L} z(x) d x / L$ ( $L$ is the length of the nanotube). Their dynamics can be described by Eq. (4) unless the amplitude of the nanotube oscillations exceeds its diameter by several orders of magnitude.

The current through the left junction ${ }^{17}$ is

$$
I(t)=e \sum_{j}\left[\Gamma_{j \rightarrow j+1}^{\mathrm{L}}(t)-\Gamma_{j \rightarrow j-1}^{\mathrm{L}}(t)\right] \rho_{j}(t) .
$$

We are interested in the current averaged over a time interval $\tau$ much larger than the characteristic period $T_{0}$ of the island oscillations: $\bar{I}=\int_{-\tau}^{\tau} I(t, z(t)) d t /(2 \tau), \tau \rightarrow \infty$.

The typical frequency of micromechanical oscillations is $\omega_{0} \sim 100 \mathrm{MHz}$. If electrons tunnel through the SET with a similar frequency, the current will be of the order of $I$ $\sim e \omega_{0} \sim 10^{-11}$ A. However, $I$ in a typical SET is usually several orders of magnitude bigger, which allows for some simplifications. In general, a state of the SET is characterized by the parameters $z, \dot{z}$, and $n$, which have the probability distribution $P(z, \dot{z}, n ; t)$. During the oscillation period $T_{0}$ many electrons go through the SET, hence we can approximate $P(z, \dot{z}, n ; t) \approx \rho_{n}(z, t) P(z, \dot{z} ; t)$. This "adiabatic" assumption justifies the use of Eqs. (1)-(4). With the same accuracy we can neglect the time derivative in the master Eq. (3) in the calculation of the average current. Then, all the methods used to calculate the current in standard SETs are applicable to the case with the oscillating island. ${ }^{17}$ Averaging the current over time can be replaced by averaging over $P(z)$, the density of the probability distribution for the deviation $z$, i.e., $\bar{I}=\int P(z) I(z) d z$. If the island oscillations are thermally acti- vated, $P(z) \propto \exp \left(-z^{2} / 2\left\langle z^{2}\right\rangle_{T}\right)$, where $\left\langle z^{2}\right\rangle_{T}=k_{B} T / m \omega_{0}^{2}$. If the island oscillates harmonically, $z(t)=z_{0} \sin (\omega t)$, then $P(z)$ $=1 /\left[\pi \sqrt{1-\left(z / z_{0}\right)^{2}}\right]$ for $|z|<z_{0}$ and zero otherwise. In these expressions, the driving terms $\sim f$ in Eq. (4) that couple the current in the SET with its mechanical degrees of freedom were neglected. This term is usually much smaller than $\omega_{0}^{2} z$ on the left-hand side of Eq. (4) (e.g., for the SET parameters in recent experiments, see Ref. 10): The small parameter is $z_{0} \max _{i} \partial_{z} \ln C_{i}$. The driving terms may become important, e.g., when an ac bias near the resonance frequency $\omega_{0}$ is applied to the terminal(s) of the SET. The stochastic tunneling of the SET also transfers energy to the oscillator. A sufficient dissipation can be estimated using energy balance equations. ${ }^{21}$ For typical parameters, we obtain an upper limit for the quality factor $Q<\left(\Gamma / \omega_{0}\right)\left(E_{0} / E_{c}\right)^{2} /\left(z_{0} \partial_{z} \ln C_{g}\right)^{2} \approx 10^{6}$, where $\Gamma$ is the tunneling rate and $E_{0}$ is the energy of the oscillator. Thus, our calculations are relevant for transport experiments in current nanomechanical systems.

In general, the current and noise in a SET cannot be calculated analytically for arbitrary transport voltages ${ }^{22}$ even when the island is static. Analytical progress can be made if we restrict ourself to the case of small driving voltages near the onset voltage, and temperatures much below the charging energy $e^{2} / C_{\Sigma}$, i.e., $\gamma(\epsilon) \approx \theta(\epsilon)$ in Eq. (2). In the case of a static island, the performance of the SET as a transistor and electrometer reaches an optimum in this regime. ${ }^{22}$ In this region, the transport characteristics of the SET are also most sensitive to mechanical oscillations of the island, so this regime is the most interesting for us. Only two states of the island have to be taken into account; the probability $\rho$ has only two nonzero values $\rho_{n}, \rho_{n+1} .{ }^{22}$ If $V_{\mathrm{L}}<V_{\mathrm{R}}$ an electron enters the island with the rate $\Gamma_{n \rightarrow n+1}^{\mathrm{L}}(z)$ from the left lead and goes away with the rate $\Gamma_{n+1 \rightarrow n}^{R}(z)$ into the right lead. The average current will be

$$
\bar{I}=\int d z P(z) \frac{e \Gamma_{n \rightarrow n+1}^{\mathrm{L}}(z) \Gamma_{n+1 \rightarrow n}^{\mathrm{R}}(z)}{\Gamma_{n \rightarrow n+1}^{\mathrm{L}}(z)+\Gamma_{n+1 \rightarrow n}^{\mathrm{R}}(z)},
$$

where $C_{g}^{(0)} \equiv C_{g}(z=0)$, and $n=\left[-C_{g}^{(0)} V_{g} / e\right]$, here $[\ldots]$ means the integer part. Assuming that the capacitances depend only weakly on $z$, Eq. (6) can be expanded with respect to $z$. To proceed, we define

$$
J(z)=\frac{1}{e} \frac{\Delta E_{n \rightarrow n+1}^{\mathrm{L}} \Delta E_{n+1 \rightarrow n}^{\mathrm{R}}}{R_{\mathrm{R}} \Delta E_{n \rightarrow n+1}^{\mathrm{L}}+R_{\mathrm{L}} \Delta E_{n+1 \rightarrow n}^{\mathrm{R}}} .
$$

Using $\Delta E_{n+1 \rightarrow n}^{\mathrm{R}}=e\left(V_{\mathrm{R}}-V_{\mathrm{L}}\right)-\Delta E_{n \rightarrow n+1}^{\mathrm{L}}$ and defining $z_{1}, z_{2}$ to be the roots of the equations $\Delta E_{n \rightarrow n+1}^{\mathrm{L}}=0$ and $\Delta E_{n+1 \rightarrow n}^{R}=0$, we get from Eq. (6)

$$
\begin{aligned}
\bar{I} \approx & \int_{\min \left(z_{1}, z_{2}\right)}^{\max \left(z_{1}, z_{2}\right)} d z P(z)\left[J(0)+z\left(\left.\partial_{z} J(z)\right|_{z \rightarrow 0}\right)\right. \\
& \left.+z^{2} \frac{1}{2}\left(\left.\partial_{z}^{2} J(z)\right|_{z \rightarrow 0}\right)\right] .
\end{aligned}
$$

This formula is valid also for $V_{\mathrm{L}}>V_{\mathrm{R}}$. Using Eq. (1) we find $z_{2}-z_{1} \approx e\left(V_{\mathrm{R}}-V_{\mathrm{L}}\right) /\left.\partial_{z} \epsilon_{n}\right|_{z \rightarrow 0}$. Thus, if $z_{0}$ is a characteristic amplitude of island oscillations then it is natural to define the voltage scale 


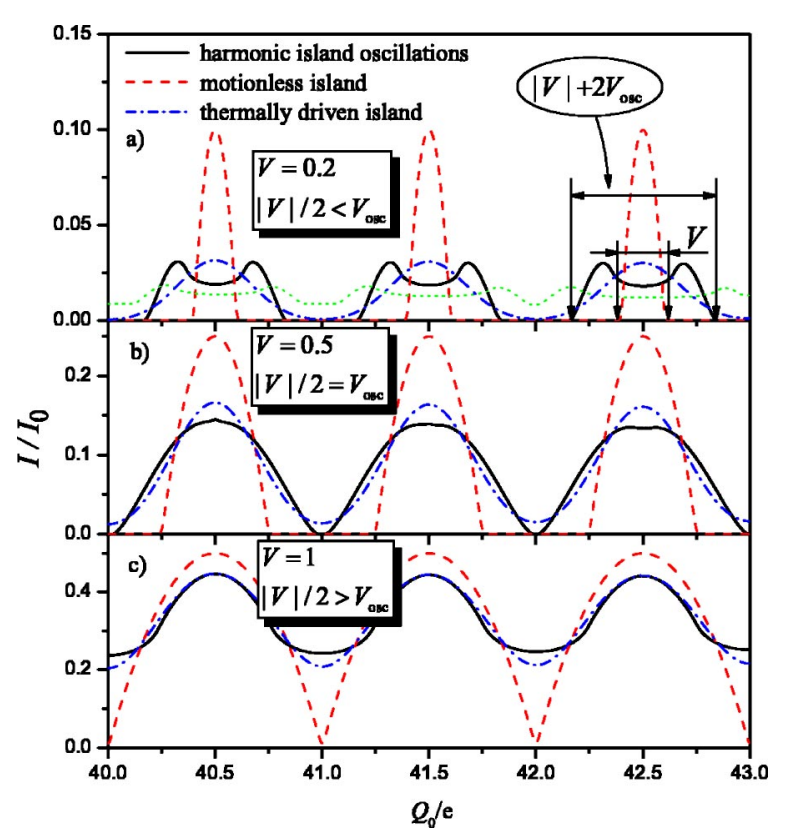

FIG. 2. (Color online) Current gate-charge characteristics for a symmetric $\operatorname{SET}\left(R_{\mathrm{L}}=R_{\mathrm{R}}\right)$ with $C_{g} \gg C_{\mathrm{L}, \mathrm{R}}, V_{\mathrm{L}}=-V_{\mathrm{R}}=V / 2, Q_{0}$ $=-C_{g}^{(0)} V_{g}$. (a) $V=0.2,\left(|V| / 2<V_{\text {osc }}\right)$, (b) $V=0.5,\left(|V| / 2 \approx V_{\text {osc }}\right)$, (c) $V=1, \quad\left(|V| / 2>V_{\text {osc }}\right)$. The voltage is measured in units of $|e| / 2 C_{\Sigma}(z=0)$, the current in units of $I_{0}=\left(e / C_{g}^{(0)}\right) /\left(R_{\mathrm{L}}+R_{\mathrm{R}}\right)$. The dashed curve corresponds to a static island, the solid curve to a harmonically oscillating island, $z=z_{0} \sin \left(\omega_{0} t\right) ; z_{0}\left(\partial_{z} C_{g}\right) / C_{g}=5.6$ $\times 10^{-3}$ (this is typical for SETs where the island is a nanotube; ${ }^{10}$ then $z_{0} \approx 5 r$, where $r$ is a typical nanotube diameter). The dotted curve in Fig. 1(a) illustrates what happens if $V_{\text {osc }} /\left(|e| / 2 C_{g}^{(0)}\right)$ $=5>1$ and Eq. (8) is not valid. The dash-dotted curves correspond to the case of thermal motion; the thermal average $\left\langle z^{2}\right\rangle_{T}$ $\equiv k_{B} T / m \omega_{0}^{2}$ is chosen to be equal $\left\langle\left[z_{0} \sin \left(\omega_{0} t\right)\right]^{2}\right\rangle_{t}=z_{0}^{2} / 2$. The integer part of $Q_{0} / e$ is the number of electrons on the island in the static regime when $V_{\mathrm{L}}=V_{\mathrm{R}}=0$. The curves are periodic in the static case, but not if the island oscillates. The areas under the peaks in the static and in the dynamical cases are the same.

$$
V_{\mathrm{osc}}=\frac{z_{0}}{e}\left(\left.\partial_{z} \epsilon_{n}\right|_{z \rightarrow 0}\right)
$$

In the limiting case $C_{\mathrm{L}}, C_{\mathrm{R}} \ll C_{g}^{(0)}$,

$$
V_{\mathrm{osc}}=\frac{\left.z_{0} \partial_{z} C_{g}\right|_{z \rightarrow 0}}{\left[C_{g}^{(0)}\right]^{2}} e(n+1 / 2) .
$$

Equation (8) is valid if $V_{\mathrm{osc}}<e / C_{\Sigma}$. If the driving voltages applied to the SET terminals are much larger than $V_{\text {osc }}$, the integration limits in Eq. (8) can be extended to infinity because they far exceed $z_{0}$, the scale of decay of $P(z)$. The second term in Eq. (8) vanishes and

$$
\bar{I}=I(z=0)+\left.\frac{1}{2}\left\langle z^{2}\right\rangle \frac{\partial^{2}}{\partial z^{2}} I(z)\right|_{z \rightarrow 0},
$$

where $\left\langle z^{2}\right\rangle=\int P(z) z^{2} d z$. The first term in Eq. (11) is the current for a static island. If the driving voltages applied to SET terminals are smaller than $V_{\text {osc }}$ then the second term (linear in $z$ ) in Eq. (8) does not vanish; In this regime the current-

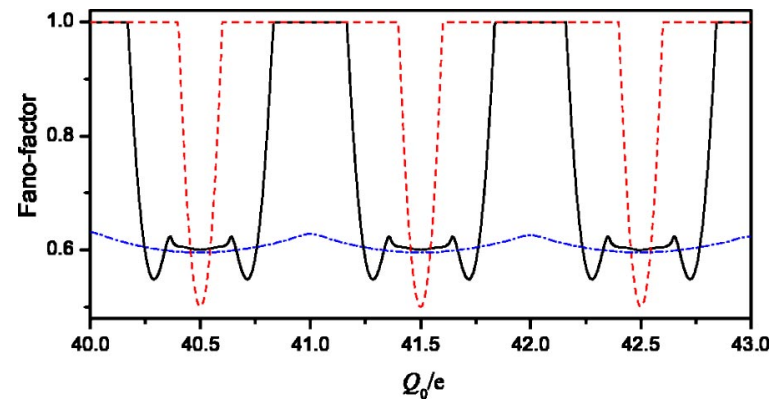

FIG. 3. (Color online) Fano factor for $V<V_{\text {osc }}$. The dashed line corresponds to the static case, the solid line to the harmonically oscillating island, and the dash-dotted line to the thermally driven island. The average square amplitude of the island oscillations are equal for the harmonically oscillating island and the case of thermal equilibrium.

voltage characteristics is strongly influenced by island oscillations. The small parameter in the expansion Eq. (8) is $z_{0} \partial_{z} \ln C_{g}$. The second term in Eq. (11) is of second order in this parameter. The oscillations of the island will modify the Coulomb gap in the $I-V$ characteristics within a voltage band of width of order $V_{\text {osc }}$.

The $I-V_{g}$ characteristics of a symmetric SET $\left(R_{\mathrm{L}}=R_{\mathrm{R}}\right.$, $C_{\mathrm{L}}=C_{R} \ll C_{g}$ ) with an oscillating island is shown in Fig. 2. The dashed curves correspond to the case of the static island. The solid and dash-dotted curves show the case of a harmonically oscillating island and an island subject to thermal equilibrium fluctuations, respectively. Figures 2(a)-2(c) also show how the $I-V_{g}$ characteristics change when the driving voltage $V$ is smaller, of the order of, or larger than $V_{\text {osc }}$. Within each peak of the curves, $n$ is constant,therefore $V_{\text {osc }}$ is also constant within the peak. The most interesting case is shown in the first panel of Fig. 2(a). For the harmonically oscillating island, the peaks split and their width becomes larger with the characteristic scale $V_{\text {osc }}$; when the island moves due to thermal activation the peaks broaden with $V_{\text {osc }}$ but do not split. Thus, the type of motion of the island leaves a characteristic trace in the $I-V_{g}$ plot. Equation (8) describes the $I-V_{g}$ characteristics well if the peaks do not overlap, like in Figs. 2(a) and 2(b). It follows from Eq. (6) that the areas under the peaks in the static and dynamic cases are equal. The $I-V_{g}$ characteristics are periodic in the static case, but not periodic for an oscillating island because $V_{\text {osc }}$ changes from peak to peak, see, e.g., Eq. (10). For this graph, we used the parameters of the nanotube model of the island (see Ref. 10); we chose, e.g, $C_{g}(z)=L / 2 \ln [2(R-z) / r]$, where $r$ $=0.65 \mathrm{~nm}$ and $L=500 \mathrm{~nm}$ are the nanotube radius and length, and $R=100 \mathrm{~nm}$ is the distance to the gate. If $C_{\mathrm{L}}, C_{\mathrm{R}}$, and $C_{g}$ are of the same order, the $I-V_{g}$ characteristics is qualitatively similar to what is shown in Fig. 2. For an asymmetric junction, $R_{\mathrm{R}} \neq R_{\mathrm{L}}$, the peaks in Fig. 2 become asymmetric as well (not shown).

We finally discuss the current noise in a SET with a moving island. When the island oscillates, the irreducible currentcurrent correlator $S(\tau, \Theta)=\left\langle\left\langle I_{L}(\Theta+\tau / 2) I_{L}(\Theta-\tau / 2)\right\rangle\right\rangle$ depends on both $\tau$ and $\Theta$ (rather than only on $\tau$ as in the case of the static island). However, since the charging events in the SET are correlated on time scales much shorter than the 
period of the island oscillations, the dependence of $S$ on $\tau$ is much stronger than on $\Theta$, and the zero-frequency noise can be found as $\int S(\tau, \Theta) d \tau$, where the bar means averaging over $\Theta$. In other words, the low-frequency noise can be calculated at a given position of the island (see, e.g., Ref. 17) and then averaged over time as it was already done for the current above. The result of this procedure is presented in Fig. 3 which shows the dependence of the Fano factor ${ }^{23}$ on $V_{g}$. Here we assumed that the driving voltage is smaller than $V_{\text {osc }}$, i.e., the system is in the regime in which the influence of the oscillations of the island on the transport properties of the SET is maximal. For harmonic oscillations of the island the dips in the Fano factor split; the scale of the splitting is $V_{\text {osc }}$. In contrast to that, the dips are washed out by thermal equilibrium oscillations of the island. In the region between the peaks, the Fano factor is strongly suppressed for a thermally vibrating island.

So far we assumed that the noise frequency is much below the frequency of the island oscillations, $\omega_{0}$. We now consider the noise spectral density, i.e., the Fourier transform $\overline{S(\omega, \Theta)}$ of $\overline{S(\tau, \Theta)}$. If $\omega$ approaches $\omega_{0}$, the correction to the noise from the motion of the island is $\sim\left(\left.\partial_{z} I\right|_{z \rightarrow 0}\right)^{2} \overline{z^{2}}{ }_{\omega}$, where $\bar{z}_{\omega}^{2}=\int d \tau \exp (i \omega \tau) \overline{z(\Theta+\tau / 2) z(\Theta-\tau / 2)}$ is the spectral density of the deviation $z$ at frequency $\omega$. It has a $\delta$ peak at $\omega_{0}$ if the island oscillates harmonically. In contrast, if the island moves due to thermal activation, $\bar{z}_{\omega}^{2}=2 \eta k_{B} T /\left\{m\left[\left(\omega^{2}-\omega_{0}^{2}\right)^{2}\right.\right.$ $\left.\left.+\omega^{2} \eta^{2}\right]\right\},{ }^{20}$ the noise peak has a width of the order of the oscillation damping factor $\eta,{ }^{24}$ see Eq. (4). ${ }^{24,25}$ Thus, measuring the noise spectrum allows to find the frequency of island oscillations and gives information on the nature of the oscillations. $^{26}$

In conclusion, we have discussed how vibrations of the island in a SET change its transport properties, viz., the average current, the Fano factor, and the noise spectral density. The transport characteristics of the SET can be used to determine the nature of island motion, in particular, to estimate the amplitude and frequency of its oscillations.

The authors would like to thank Ya. M. Blanter and C. Schönenberger for stimulating discussions. This work was financially supported by RFBR and the Russian Ministry of Sciences (N.M.C.), by the Swiss NSF, and the NCCR Nanoscience.
${ }^{1}$ A. N. Cleland, Foundations of Nanomechanics (Springer, Heidelberg, 2002).

${ }^{2}$ A. N. Cleland and M. L. Roukes, Nature (London) 392, 160 (1998)

${ }^{3}$ L. Y. Gorelik, A. Isacsson, M. V. Voinova, B. Kasemo, R. I. Shekhter, and M. Jonson, Phys. Rev. Lett. 80, 4526 (1998).

${ }^{4}$ A. Erbe, R. H. Blick, A. Tilke, A. Kriele, and J. P. Kotthaus, Appl. Phys. Lett. 73, 3751 (1998); A. Erbe, C. Weiss, W. Zwerger, and R. H. Blick, Phys. Rev. Lett. 87, 096106 (2001).

${ }^{5}$ C. Weiss and W. Zwerger, Europhys. Lett. 47, 97 (1999).

${ }^{6}$ T. Novotný, A. Donarini, C. Flindt, and A.-P. Jauho, Phys. Rev. Lett. 92, 248302 (2004); C. Flindt, T. Novotny, and A.-P. Jauho, cond-mat/0405512 (unpublished).

${ }^{7}$ A. Isacsson and T. Nord, Europhys. Lett. 66, 708 (2004).

${ }^{8}$ F. Pistolesi, Phys. Rev. B 69, 245409 (2004).

${ }^{9}$ J. Cao, Q. Wang, D. Wang, and H. Dai, cond-mat/0312239 (unpublished).

${ }^{10}$ S. Sapmaz, Ya. M. Blanter, L. Gurevich, and H. S. J. van der Zant, Phys. Rev. B 67, 235414 (2003).

${ }^{11}$ A. D. Armour, M. P. Blencowe, and Y. Zhang, Phys. Rev. B 69, 125313 (2004).

${ }^{12}$ A. Mitra, I. Aleiner, and A. J. Millis, Phys. Rev. B 69, 245302 (2004).

${ }^{13}$ A. V. Shytov, L. S. Levitov, and C. W. J. Beenakker, Phys. Rev. Lett. 88, 228303 (2002); M. Kindermann and C. W. J. Beenakker, Phys. Rev. B 66, 224106 (2002).
${ }^{14}$ B. Babic, J. Furer, S. Sahoo, Sh. Farhangfar, and C. Schönenberger, Nano Lett. 3, 1577 (2003).

${ }^{15}$ C. W. J. Beenakker, Phys. Rev. B 44, 1646 (1991).

${ }^{16}$ D. V. Averin, A. N. Korotkov, and K. K. Likharev, Phys. Rev. B 44, 6199 (1991).

${ }^{17}$ S. Hershfield, J. H. Davies, P. Hyldgaard, C. J. Stanton, and J. W. Wilkins, Phys. Rev. B 47, 1967 (1993).

${ }^{18}$ C. Bruder and H. Schoeller, Phys. Rev. Lett. 72, 1076 (1994).

${ }^{19}$ D. A. Bagrets and Yu. V. Nazarov, Phys. Rev. B 67, 085316 (2003).

${ }^{20}$ L. D. Landau and E. M. Lifshits, in Course in Theoretical Physics, Vol. 5 (Pergamon Press, Oxford, 1996).

${ }^{21}$ Ya. M. Blanter, O. Usmani, and Yu. V. Nazarov, Phys. Rev. Lett. 93, 136802 (2004).

${ }^{22}$ G. L. Ingold and Yu. V. Nazarov, in Single Charge Tunneling, edited by H. Grabert and M. H. Devoret, NATO ASI Series B Vol. 294 (Plenum Press, New York, 1992).

${ }^{23}$ Ya. M. Blanter and M. Büttiker, Phys. Rep. 336, 1 (2000).

${ }^{24}$ The correction to the low-frequency noise is negligible because of the presence of the small parameter $\left.\partial_{z} I\right|_{z \rightarrow 0} \sim z_{0} \partial_{z} \ln C_{g}$.

${ }^{25}$ A numerical study of a similar effect was done by A. D. Armour, Phys. Rev. B 70, 165315 ( 2004).

${ }^{26}$ The current noise limits the sensitivity of the device as a displacement detector, see D. Mozyrsky, I. Martin, and M. B. Hastings, Phys. Rev. Lett. 92, 018303 (2004). 